\title{
Mutation of the nuclear pore complex component, aladin1, disrupts asymmetric cell division in Zea mays (maize)
}

\author{
Norman B. Best (D) , 1,2,3,* Charles Addo-Quaye (ID , 3,4 Bong-Suk Kim, ${ }^{5}$ Clifford F. Weil, ${ }^{6,7}$ Burkhard Schulz, ${ }^{2}$ Guri Johal (DD , 5,7 and
} Brian P. Dilkes, ${ }^{3,7, *}$

${ }^{1}$ Plant Genetics Research Unit, USDA, Agriculture Research Service, Columbia, MO 65211, USA

${ }^{2}$ Department of Horticulture \& Landscape Architecture, Purdue University, West Lafayette, IN 47907, USA

${ }^{3}$ Department of Biochemistry, Purdue University, West Lafayette, IN 47907, USA

${ }^{4}$ Natural Sciences and Mathematics Division, Lewis-Clark State College, Lewiston, ID 83501, USA

${ }^{5}$ Department of Botany and Plant Pathology, Purdue University, West Lafayette, IN 47907, USA

${ }^{6}$ Department of Agronomy, Purdue University, West Lafayette, IN 47907, USA

${ }^{7}$ Center for Plant Biology, Purdue University, West Lafayette, IN 47907, USA

*Corresponding author: Department of Biochemistry, Purdue University, Whistler Building Room B036B, West Lafayette, IN 47907, USA. bdilkes@purdue.edu (B.P.D.); University of Missouri, USDA-ARS, 203 Curtis Hall, Columbia, MO 65211, USA. norman.best@usda.gov (N.B.B.)

\begin{abstract}
The nuclear pore complex (NPC) regulates the movement of macromolecules between the nucleus and cytoplasm. Dysfunction of many components of the NPC results in human genetic diseases, including triple A syndrome (AAAS) as a result of mutations in ALADIN. Here, we report a nonsense mutation in the maize ortholog, aladin1 (ali1-1), at the orthologous amino acid residue of an AAAS allele from humans, alters plant stature, tassel architecture, and asymmetric divisions of subsidiary mother cells (SMCs). Crosses with the stronger nonsense allele ali1-2 identified complex allele interactions for plant height and aberrant SMC division. RNA-seq analysis of the ali1-1 mutant identified compensatory transcript accumulation for other NPC components as well as gene expression consequences consistent with conservation of ALADIN1 functions between humans and maize. These findings demonstrate that ALADIN1 is necessary for normal plant development, shoot architecture, and asymmetric cell division in maize.
\end{abstract}

Keywords: nuclear pore complex; asymmetric cell division; stomata; subsidiary cells; maize

\section{Introduction}

The nuclear pore complex (NPC) is a multi-protein complex that is involved in regulating the movement of macromolecules into and out of the nucleus. The NPC also plays important roles in nuclear assembly during cell division (Rasala et al. 2006). NPC proteins assemble in groups of eight, as spokes, around the opening of the pore to control transport between the nucleus and cytoplasm (Reichelt et al. 1990; Lin et al. 2016). Components of the NPC are conserved across many eukaryotic species, including mammals (Cronshaw et al. 2002), plants (Tamura et al. 2010; Tamura and Hara-Nishimura 2013), fungi (Liu et al. 2009; Stuwe et al. 2015), and yeast (Rout et al. 2000). Dysfunction and/ or mutation of the genes encoding NPC components has drastic effects on growth, development, and survival of eukaryotic species. In humans, genetic mutations of NPC members have been linked to a number of diseases and illnesses, including different types of cancer, neurological diseases, and autoimmune diseases (Roth and Wiermer 2012; Nofrini et al. 2016; Sakuma and D’Angelo 2017).

The structure and conserved components of the NPC of plants have been identified (Neumann et al. 2006; Fiserova et al. 2009; Tamura et al. 2010; Tamura and Hara-Nishimura, 2013).
Genetic studies have functionally characterized a few NPC members in plant species. The Arabidopsis NPC member, AtMOS7/Nup88, was shown to regulate the nuclear concentrations of a subset of defense proteins, but other proteins tested were not affected in Atmos7 mutants (Cheng et al. 2009). Additional forward and reverse genetic approaches in plant species; primarily Arabidopsis, tobacco, and Lotus japonica, have further characterized the function and role of NPC members in plant defense, plant symbioses, and responses to abiotic stress (Zhang and Li 2005; Dong et al. 2006; Cheng et al. 2009; Roth and Wiermer 2012; Wiermer et al. 2012; Binder and Parniske 2013). To date, no member of the NPC has been functionally characterized in Zea mays (maize). It is unclear to what extent the specific function of NPC members can be inferred from phylogeny. In comparisons between mice and human, mutation of WD-40 repeat containing NPC member ALADIN did not produce the same phenotypic outcomes (Huebner et al. 2006). It is unclear whether such species-specific phenotypic impacts will characterize NPC functions in plants.

Here, we describe the first characterization of an NPC mutant in maize and the first description of an ALADIN mutant in plants. The aladin1-1 (ali1-1) mutant was identified in an ethyl methanesulfonate (EMS) screen as a short plant with unusual tassel 
architecture and defective subsidiary cell asymmetric division in developing stomata. The ali1-1 mutant results in a weak allele and crosses with a null allele identified allelic dosage effects of the ali1 gene, demonstrating that ali1-1 was haploinsufficient for maintaining normal development especially after the phase change. RNA-sequencing identified a compensatory increase in transcripts encoding other NPC members in ali1-1, even during juvenile growth before the defects in subsidiary mother cell (SMC) asymmetric division were visible. These results implicate this gene in cell division control and NPC homeostasis that may also be true for humans and contribute to AAAS disease.

\section{Materials and methods Plant material and growth conditions}

In the summer of 2010, the ali1-1 mutant was identified in a B73 background EMS population as M3 generation plants segregating in the line 04IAB73PS007D8 (Weil 2009). The ali1-2 allele was created by an EMS-targeted mutagenesis approach. B73 pollen was incubated with $6.48 \mathrm{mM}$ EMS (Sigma-Aldrich, St. Louis, MO, USA) in paraffin oil (Sigma-Aldrich, St. Louis, MO, USA) for 35 mins then squirted onto 205 ali1-1/ali1-1 ears using a $40 \mathrm{ml}$ squeezable bottle (Hobby Lobby, Oklahoma City, OK, USA). 10,000 M1 seeds were screened in the field in the summer of 2015 in West Lafayette, IN for phenotypes similar to ali1-1. A total of 13 plants resembling ali1-1 were identified. One of the twelve was heterozygous for the causative single nucleotide polymorphism (SNP) in ali1-1. This plant was backcrossed to ali1-1/ali1-1 and B73 to propagate the ali1-2 allele.

To map the ali1 gene, an F2 population was created by crossing Mo17 with ali1-1/ali1-1 pollen and self-pollinated the F1 generation. The F2 progeny was planted in the field to collect tissue from selected wild-type and ali1-1 phenotypical plants. For RNASeq analysis on juvenile plants, wild-type and ali1-1/ali1-1 were grown in the greenhouse in trays with a 2:1 mixture of peat germinating mix (Conrad Fafard Inc., Agawam, MA, USA) to Turface MVP (Profile Products LLC, Buffalo Grove, IL, USA) and the SAM and surrounding tissue were harvested 15 days after planting (DAP). Total plant height, stomatal index, stomatal density, and percent aberrant stomata measurements of the 8th leaf were collected from plants grown in the field in the summer of 2016 in West Lafayette, IN. Plant height, internode length, tassel length, and rachis length of B73 and ali1-1 were measured on field-grown plants in the summer of 2013. The $+/+,+$ /ali1-1, +/ali1-2, ali1-1/ ali1-1, ali1-1/ali1-2, and ali1-2/ali1-2 plants used for total plant photographs and epidermal peel photographs were grown in the Purdue Horticulture Plant Growth Facility in the spring of 2016 and spring of 2017, respectively. The nine individual BC1F2 plants used for DNA sequencing were grown in the greenhouse in the spring of 2014. The temperature settings were $27^{\circ} \mathrm{C}$ (day) and $21^{\circ} \mathrm{C}$ (night) with a 16 hours day length provided by supplemental lighting. Plants were grown in two-gallon pots in soilless media as previously described.

\section{Phylogenetic analysis}

Phylogenetic analysis was performed by obtaining protein homolog coding sequences of GRMZM2G180205 through Phytozome version 10.2.2 (Goodstein et al. 2012) using the Inparanoid method (O'Brien et al. 2005) with a Dual Affine Smith-Waterman score greater than 1250 and sequence similarity of greater than 50\%, resulting in 37 sequences from 32 taxa. Sequences were downloaded and a phylogenetic consensus tree was obtained as previously described in Best et al. (2016). The N-terminus sequence was trimmed from species Manihot esculenta (cassava4_1.026236m), Malus domestica (MDP0000250771), and Solanum tuberosum (PGSC0003DMG400017881) due to incorrect start sites based upon alignment similarities.

\section{Sequencing and linkage analysis of ali1 locus}

To determine the DNA sequence of wild-type, ali1-1/ali1-1, ali1-1/ ali1-2, and ali1-2/ali1-2, total DNA was extracted and amplified using the primers described in Supplementary Table S1. PCR products were sequenced (Psomagen Inc., MD, USA) using the Sanger method (Sanger et al. 1977). Restriction fragment length polymorphism genotyping of ali1-1 and ali1-2 alleles was performed using the derived Cleaved Amplified Polymorphic Sequences (dCAPS) method (Neff et al. 1998) and separated on a $3 \%$ agarose gel in Tris-Acetate-EDTA to observe size differences. For the ali1-1 dCAPS genotyping, polymerase chain reaction (PCR) was carried out using primers DCali-1_FOR and ZmALI_REV+ 4119 (Supplementary Table S1). The amplified DNA was incubated with the restriction endonuclease TaqI (New England Biolabs; Ipswich, MA, USA) resulting in an uncut wild-type band of approximately $140 \mathrm{bp}$ and the ali1-1 mutant amplicon was cleaved to $115 \mathrm{bp}$ (Supplementary Figure S1). For the ali1-2 genotyping, amplification was carried out using primers DCali-2_FOR and ZmALI_REV + 3243 (Supplementary Table S1) and the uncut wild-type band was about $340 \mathrm{bp}$ and the ali1-2 mutant band was 320 bp following cleavage by the restriction endonuclease HindIII (New England Biolabs; Ipswich, MA, USA). Digestion of the ali1-2 fragments was incomplete and as a result, heterozygous and homozygous ali1-2 plants could not be distinguished using this molecular marker (data not shown). M37W was crossed as ear parent with ali1-1/ali1-1 pollen. F1 plants were selfed and F2 generation ali1-1/ali1-1 plants were phenotyped and tissue was collected for genotyping by the dCAPS method in the summer of 2015. Linkage of ali1-1 to the rp1 locus (AC152495.1_FG002) on maize chromosome 10 was determined by crossing ali1-1/ali1-1 ears with Rp1-D21/+ pollen (backcrossed 8 generations to B73) and then backcrossing the F1 Rp1-D21 phenotype plants with ali11/ali1-1 as ear parents (Pryor 1993). The BC1 F1 generation was phenotyped for both the Rp1-D21 and ali1-1 phenotypes in the field during the summer of 2016.

\section{Bulked segregant analysis and RNA sequencing}

For bulked segregant analysis (Michelmore et al. 1991) of SNP cosegregation with ali1-1 and mRNA accumulation differences, total RNA was extracted (Eggermont et al. 1996) from 2 replicates of 60 wild-type and 60 ali1-1/ali1-1 tassel stem punches at the location of the lowest primary tassel branch before tassel emergence. Samples were taken from F2 individuals derived by selfing the F1 progeny from a cross of Mo17 ears pollinated with ali1-1/ali1-1. Nonstranded cDNA libraries were prepared with a TruSeq RNA sample preparation kit v2 and sequenced on a HiSeq 2000 (Illumina, San Diego, CA, USA) using SBS v3-HS reagents for paired-end sequencing $(2 \times 100 \mathrm{bp})$. Raw reads were quality filtered to remove reads with less than 20 quality score and adapters were clipped using Trimmomatic (version 0.22; Bolger et al. 2014) and fastx_clipper as a part of the FASTX-Toolkit (version 0.0.13) (http://hannonlab.cshl.edu/fastx_toolkit/). Filtered reads were aligned to the B73 reference genome (version 3.30) using Bowtie2 (version 2.2.8; Langmead and Salzberg 2012). Total reads and alignment rate are shown in Supplementary Table S2. Polymorphisms between Mo17 and B73 were used to map the causative mutation in ali1-1 (Supplementary File S1). Single-nucleotide polymorphism positions were identified by aligning 
Mo17 Illumina sequence to the B73 reference genome and calling SNPs by SAMtools (version 1.3.1; Li et al. 2009) "mpileup" command. Only SNPs that were homozygous nonB73 reference, had reads on forward and reverse strands, and had less than 12 reads aligning to respective position were retained and used as SNPs for distinguishing sequencing reads from B73 and Mo17 chromosomes.

To map the causative mutation in ali1-1, B73, and Mo17 allele frequencies were determined from the ali1-1/ali1-1 and wild-type samples and plotted in 100 SNP bins for each maize chromosome (Supplementary Figures S2-S6). To identify potential causative mutations for the ali1 phenotype, SNPs that differed between the ali1-1 pools and B73 reference that were in coding sequences were identified using SAMtools mpileup command and filtered to be homozygous nonB73 reference, $\mathrm{G}$ to $\mathrm{A}$ or $\mathrm{C}$ to $\mathrm{T}$ transitions, and have reads on both forward and reverse strands at the position. Identified SNP positions were then annotated for effect on coding sequence and protein function using SnpEff (Cingolani et al. 2012). An Arabidopsis protein BLAST database was created using BLAST (version 2.6.0+) and used to align all maize protein sequences. Best hits were used to provide additional annotations to the maize genes using the TAIR10 annotations (Lamesch et al. 2012). The E-value score cutoff was set at $10^{-5}$. Therefore, if the best BLASTP result had an E-value greater than $10^{-5}$, the result was not included for annotation and these columns are denoted with an asterisk.

To identify differential mRNA accumulation between mutant and wild-type samples (Supplementary Files S2 and S3), reads were aligned to B73 reference genome (version 3.30) using Bowtie2 (version 2.2.8) (Langmead and Salzberg 2012). Counts tables were created in HTseq (version 0.6.1) (Anders et al. 2015) and passed to DESeq2 (R-bioconductor; version 3.3.0) (Love et al. 2014). Differential expression between wild-type and ali1-1 samples were assessed using the "nbinomLRT" setting with "parametric" dispersion. Additional annotation of maize differentially expressed genes was conducted by comparing Arabidopsis (version TAIR10) and maize (version 3.30) proteomes using BLASTP (Tatusova and Madden 1999). Genes were annotated as previously described.

\section{DNA sequencing of ali1 mutants}

DNA was extracted from nine individual ali1-1/ali1-1 plants, sonicated, and were libraries constructed using the Illumina TruSeq DNA PCR-free LT Library Preparation protocol. Paired-end $100 \mathrm{bp}$ reads were generated on an Illumina HiSeq 2500 using SBS v3-HS reagents. Sequencing statistics for the nine individual ali1-1/ali11; B73 BC1 plants are described in Supplementary Table S10. The nine fastq files were concatenated together for downstream analysis. Reads were mapped to the B73 reference genome (version 3.30) using BWA (version 0.7.12) (Li and Durbin 2009). SNPs in coding sequence between aligned reads and the reference genome were done as previously described. Supplemental File S6 shows high-quality SNPs as called by SAMtools and annotated for coding sequence effects by SnpEff.

\section{Quantification of stomatal phenotypes}

Epidermal imprints and peels were collected from field-grown B73 (+/+), +/ali1-1, ali1-1/ali1-1, ali1-1/ali1-2, and ali1-2/ali1-2 plants. Epidermal cell layer imprints were produced from the widest portion of the eighth leaf when the plants were at V9 stage using ethyl-2 cyanoacrylate (Scotch SuperGlue; $3 \mathrm{M}$, Maplewood, MN, USA) applied to the leaves and then pressed onto and recovered on glass microscope slides (Thermo Fisher Scientific,
Waltham, MA, USA). Imprints of the abaxial leaf surfaces were observed by light microscopy using a UNICO H606T microscope (United Products \& Instruments Inc., Dayton, NJ, USA). The percent aberrant stomata and subsidiary cells were calculated by nonrepeatedly randomly selecting viewable areas at 40X magnification until total stomata observed per sample was greater than 500 ( $n=6$ per genotype). Stomatal density and indices were calculated by counting all the cells in 5 randomly selected viewable areas between vasculature tissues for each imprint at 20X magnification. An average was calculated from 5 viewable areas from each of 6 biological replicates per genotype. An additional set of wild-type B73 (+/+), +/ali1-1, ali1-1/ali1-1, ali1-1/ali1-2, and ali1-2/ ali1-2 plants were grown in the greenhouse and epidermal cell layer imprints were used for quantification of aberrant stomata from the fourth leaf at V5 stage and tenth leaf at V11 stage were conducted as previously stated.

Leaf epidermal peels were performed by cutting 1-cm squares from greenhouse-grown plants and fixing the tissue in $4 \%$ formaldehyde, $50 \mathrm{mM} \mathrm{KPO}_{4}, 5 \mathrm{mM}$ EDTA, and $0.2 \%$ saponin at $\mathrm{pH} 7.0$ for at least 2 hours at room temperature. Leaf tissue pieces were rinsed 3 times with $\mathrm{ddH}_{2} \mathrm{O}$ and incubated in $100 \mathrm{mM} \mathrm{Na}$-acetate, $1 \%$ cellulase, and $0.5 \%$ pectinase at $\mathrm{pH} 5.0$ for at least 2 hours at room temperature. The tissue was then rinsed with ddH2O and the abaxial leaf epidermis was peeled from the rest of the leaf and incubated in a 1:10 dilution of $0.05 \% \mathrm{TBO}$ and $10 \mathrm{mM} \mathrm{Na}$-acetate until evenly stained. Epidermal peels were viewed and photographed using an Olympus BX43 light microscope with Olympus DP80 camera (Olympus Corporation, Center Valley, PA, USA) at 40X magnification.

\section{Data availability}

Seeds of ali1-1 and ali1-2 are available at the Maize Genetics COOP. Raw sequence data is available at the Short Read Archive under BioSample accession numbers: SAMN06764664, SAMN06764861, SAMN06765376, and SAMN07187450. All supplementary figures and tables as well as files containing the processed SNP and differential expression analyses are available at figshare: https://doi. org/10.25387/g3.14273330.

\section{Results \\ Characterization and identification of the ali1 mutant}

Mutagenized progeny of the maize inbred B73 (Weil 2009) were screened in the M3 generation for reduced stature phenotypes in the field. The ali1-1 mutant was identified as semi-dwarf maize plants with reduced tassel length in a single M3 family. Shown in Figure 1 are the ali1 mutant gross morphological phenotypes. The ali1-1 mutation was inherited as a monogenic recessive trait after backcrossing to the wild-type B73 (Figure 1, A and B). Field grown ali1-1 mutants were significantly shorter than wild-type plants and exhibited a 29 or $27 \%$ reduction in plant height measured to the tip of the tassel or flag leaf collar, respectively (Figure 1, F and $\mathrm{G})$. The reduction in plant height observed in ali1-1 mutants was due to compression of the first two and last five internodes compared to wild-type siblings (Figure 1, A, B, and H). The largest suppression of internode length was observed in the internodes above the ears, which elongate primarily after phase change has occurred (Fournier and Andrieu 2000). Total tassel length of ali1-1 mutants was $40 \%$ reduced compared to wild type (Figure $1 \mathrm{I}$ ). Tassel and stem elongation were variably affected, and some tassels did not completely emerge from the leaf whorl. The rachis length, as measured from the lowest tassel branch to the tip of 

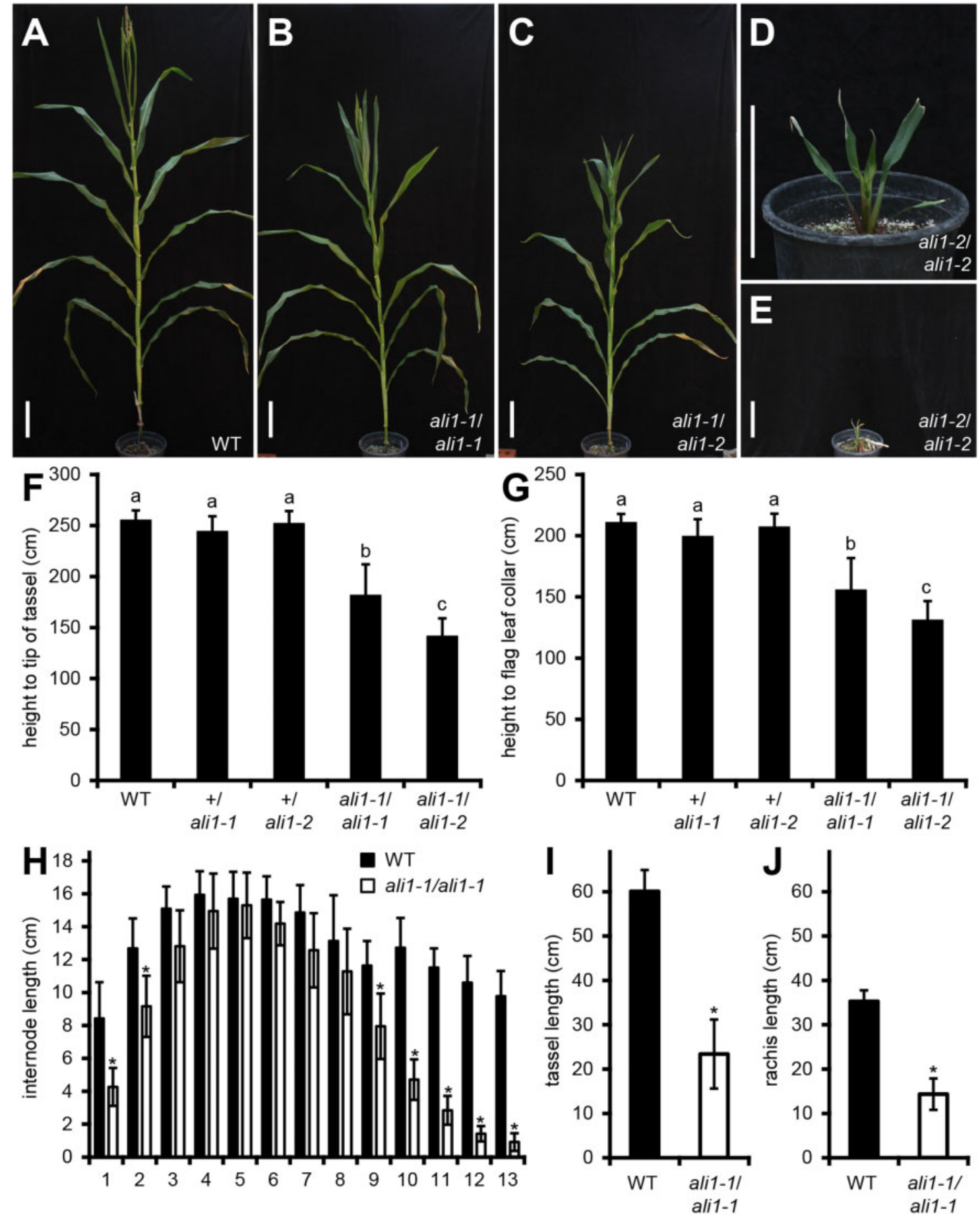

Figure 1 Morphological features of ali1 mutants. (A) Mature wild-type (+/ali1-1), (B) ali1-1/ali1 -1, (C) ali1 -1/ali1 -2, (D and E), and ali1 -2/ali1-2 plants grown in the greenhouse. (F) Total plant height measured to tip of tassel and (G) plant height measured to flag leaf collar at maturity of wild-type $(n=17),+$ /ali1-1 $(n=16),+$ /ali1-2 $(n=20)$, ali1-1/ali1-1 $(n=15)$, and ali1-1/ali1-2 $(n=14)$ grown in the field. (H) Internode lengths of field-grown wild-type $(n=25)$ and ali1-1/ali1-1 $(n=17)$ plants. Internode one indicates the internode closest to the soil. (I) Tassel length, including the peduncle and rachis, of wild-type $(n=25)$ and ali1-1/ali1-1 $(n=17)$ plants. (J) Rachis length of wild-type $(n=25)$ and ali1-1/ali1-1 $(n=17)$ plants measured from the lowest tassel branch to the tip of the rachis. (A-E) Scale bars represent $20 \mathrm{~cm}$. (F and G) Connecting letter report determined by Student's T-test with Bonferroni corrected $P$-value $(P<0.005)$ for multiple testing. $(H-J)$ asterisks indicate Student's T-test $(P<0.01)$ for comparisons between wild-type and ali1-1/ali1-1 at given internode. (F-J) Error bars are standard deviations.

the rachis, was $41 \%$ less in ali1-1 mutants as compared to wildtype siblings and tassel internodes were shortened along the entire distal axis of the tassel in the ali1-1 mutants (Figure 1J). Tassel branching was also reduced in ali1-1 mutants (Table 1). Visible phenotypes in ali1-1 mutants primarily affected adult tissues that developed after phase change, suggesting a progressive effect on plant development resulting from the ali1-1 mutation. Similar to field-grown plants, the ali1-1 mutant was significantly shorter than wild type when grown in the greenhouse in spring months of 2016 (Tables 1 and 2). By contrast, when grown in the greenhouse during the shorter days of winter 2015, ali1-1 mutants were considerably taller, possibly due to suppression of the 
Table 1 Morphometric analysis of B73, ali1-1/ali1-1, and ali1-1/ali1-2 grown in the spring of 2016 in greenhouse

\begin{tabular}{|c|c|c|c|c|c|}
\hline Genotype & Ear height $^{a}$ & flag leaf height ${ }^{a}$ & tassel height ${ }^{a}$ & tassel length $^{a}$ & tassel branches ${ }^{a}$ \\
\hline $\begin{array}{l}\text { B73 (+/+) } \\
\text { ali1-1/ali1-1 } \\
\text { ali1-1/ali1-2 }\end{array}$ & $\begin{array}{l}92.4 \pm 8.0 \mathrm{a} \\
78.8 \pm 8.3 \mathrm{a} \\
93.3 \pm 16.2 \mathrm{a}\end{array}$ & $\begin{array}{l}219.0 \pm 6.7 \mathrm{a} \\
177.8 \pm 8.2 \mathrm{~b} \\
172.4 \pm 14.4 \mathrm{~b}\end{array}$ & $\begin{array}{l}261.4 \pm 6.9 \mathrm{a} \\
211.0 \pm 7.5 \mathrm{~b} \\
194.3 \pm 14.1 \mathrm{c}\end{array}$ & $\begin{array}{l}54.6 \pm 1.8 \mathrm{a} \\
43.3 \pm 5.8 \mathrm{~b} \\
29.5 \pm 6.0 \mathrm{c}\end{array}$ & $\begin{array}{l}7.6 \pm 1.5 \mathrm{a} \\
5.4 \pm 1.7 \mathrm{ab} \\
3.8 \pm 1.3 \mathrm{~b}\end{array}$ \\
\hline
\end{tabular}

${ }^{a}$ Units are in $\mathrm{cm} \pm \mathrm{SD}$. Lowercase letters indicate connecting letter report as determined by ANOVA with posthoc analysis using the Holm-Sidak algorithm with $\mathrm{P}<0.05$.

Table 2 Plant height of ali1-1 was suppressed in the greenhouse when grown in the winter of 2015 as compared to field-grown plants

\begin{tabular}{lcc}
\hline & $n$ & flag leaf height \pm SD $^{a}$ \\
\hline B73 in greenhouse $^{b}$ & 5 & $240.8 \pm 12.28 \mathrm{a}$ \\
B73 in field $^{c}$ & 16 & $239.5 \pm 6.54 \mathrm{a}$ \\
ali1-1/ali1-1 in greenhouse $^{b}$ & 18 & $234.4 \pm 14.02 \mathrm{a}$ \\
ali1-1/ali1-1 in field $^{c}$ & 46 & $157.0 \pm 19.03 \mathrm{~b}$ \\
\hline
\end{tabular}

${ }^{a}$ Units are in $\mathrm{cm}$, lowercase letters indicate connecting letter report as determined by ANOVA with post hoc analysis using the Holm-Sidak algorithm with $P<0.05$.

${ }^{b}$ Plants were grown in greenhouse in winter of 2015

cPlants were grown in field in summer of 2015 in WL.

mutant phenotype by short days and/or low light quality and ali1-1 plants were visually indistinguishable from wild-type siblings.

The genetic map position for ali1-1 was determined by bulkedsegregant analysis of RNA-Seq data from F2 mutant and wildtype pools of ali1-1 x Mo17. Reads were mapped to the B73 reference sequence and the allele frequencies at SNP positions between Mo17 and B73 (Supplementary File S1) were scored in 100SNP bins and graphed (Supplementary Figures S2-S5). A region near the telomere of chromosome 10S approached homozygosity for B73 in the mutant pools (Supplementary Figure S6; Supplementary Files S4-S7). To obtain additional linkage information, ali1-1 was crossed to Rp1-D21/+, a hyper active allele of a NUCLEOTIDE-BINDING LEUCINE-RICH REPEAT (NLR) gene for resistance to Puccina sorghi on $10 \mathrm{~S}$ (Pryor 1993), and F1 progeny were backcrossed to ali1-1 homozygotes. In the BC1 F1, 23 recombinant individuals were recovered out of 322 progeny, demonstrating that ali1 was located $7.1 \mathrm{cM}$ from the Rp1 locus on chromosome $10 S$ (Supplementary Table S3). Genomic DNA was sequenced from ali1-1 mutants derived from nine independent ali1-1 x B73 BC1 F2 families. Within the mapped region, a single homozygous $G$ to A transition was identified which resulted in a premature stop codon in the last exon of GRMZM2G180205 (v4 gene model Zm00001d023264) removing the last 16 amino acids from the protein-coding sequence (Supplementary Figures S7A-S9, and Supplementary File S8). Perfect co-segregation between the mutant phenotype and this $\mathrm{G}$ to A transition $(n=125)$ was observed in a F2 population derived by crossing ali1-1 with the maize inbred line M37W. GRZM2G180205 encodes a WD-40 repeat protein and the ortholog of the triple A syndrome (AAAS; OMIM 231550) human disease gene, also called the ALacrimia-AchalasiaaDrenal Insufficiency Neurologic disorder1 (ALADIN1) protein, which is a component of the NPC (Supplementary Figures S7B and S9). A large-scale duplication of $\sim 20 \mathrm{~kb}$ at this locus resulted in a pseudogene annotated as GRZM2G180249 (v4 gene model Zm00001d023266) and encoding sequences similar to the latter half of the protein sequence of GRZM2G180205. All B73-derived ESTs in NCBI match the sequence of GRZM2G180205 and not GRZM2G180249 at the 10 SNPs and N-terminal deletion that distinguish the paralogs (Supplementary Table S4). The ali1-1 mutant (Exon 16, W430*) resulted in an amber stop codon within the final WD-40 domain of ALADIN1. A nonsense allele at the homologous position in the human ALADIN protein encodes a disease allele (Exon 16, W474*) that results in moderate AAAS when inherited in combination with a likely null allele, but has not been observed as a homozygote in human (Houlden et al. 2002).

\section{ali1 affects plant development and asymmetric cell division in an allelic dosage manner}

A targeted EMS mutagenesis approach was used to generate additional mutant ali1 alleles. Mutants were used as ear parents and crossed with EMS-mutagenized B73 pollen. 10,000 M1 plants were screened for the ali1-1 phenotype and mutant plants were genotyped at the ali1-1 allele by dCAPS to distinguish pollen contaminants and gynogenetic haploids (Sarkar and Coe 1966) from plants harboring novel alleles. Twelve plants displayed phenotypes reminiscent of ali1-1, but 11 were homozygous for ali1-1. One plant was heterozygous for the ali1-1 mutation and sequencing of GRMZM2G180205 isolated by PCR from DNA extracted from this plant contained a new $\mathrm{C}$-to- $\mathrm{T}$ transition at nucleotide position 2925 in exon 10 resulting in a premature stop codon that would remove the last 134 amino acids (Supplementary Figures S7A-S9). Testcrosses to ali1-1 and wild-type B73 confirmed that the new allele, ali1-2, failed to complement ali1-1, was recessive to the wildtype allele, and that ali1-1/ali1-2 heterozygotes had a phenotype intermediate to the two homozygotes (Figure 1, F and G). The ali12 homozygotes were severely reduced in height and arrest in development after producing 9-10 leaves, without developing a tassel or ear or undergoing stem elongation (Figure 1, D and E).

The mutant alleles displayed incomplete dominance to one another, unlike their recessive relationship to the wild-type allele. The ali1-1/ali1-2 heterozygotes were 55\% the height of wildtype, $77 \%$ the height of ali1-1 homozygotes (Figure 1, F and G), and were taller than ali1-2 homozygotes. In addition, unlike ali1-2 homozygotes which fail to produce any seeds, ali1-1/ali1-2 heterozygotes were fertile. Given the greater severity of the ali1-2 phenotype, this suggests that the gene product encoded by the ali1-1 allele was partially functional and dosage sensitive.

Numerous defects in leaf morphology were visible in ali1-1 and ali1-2 mutants. Some ali1-2 mutant leaves split and became necrotic at the midrib as they aged. Both ali1-1 and ali1-2 leaf surfaces were crinkly or wrinkled in appearance (Figure 1). To investigate the basis of this, epidermal peels were taken from one juvenile leaf (leaf 4) and one adult leaf (leaf 10) from greenhousegrown plants, and an adult leaf 8 from field-grown plants. In adult leaves, both ali1-1 and ali1-2 mutants had more aberrant stomatal complexes than nonmutant plants, primarily due to aberrant or a lack of SMC divisions, and ali1-2 was more severely affected than ali1-1 (Figure 2, A-D). Inheritance of this leaf trait was monogenic recessive (Figure 2, E-G). Consistent with the greater severity of defects in adult tissues, the ali1-1 mutants did not discernably alter SMC division in the juvenile fourth leaf. The more severe ali1-2 mutant, on the other hand, did affect aberrant SMC 


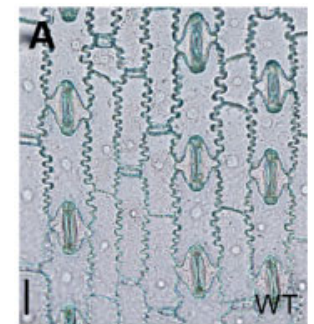

E

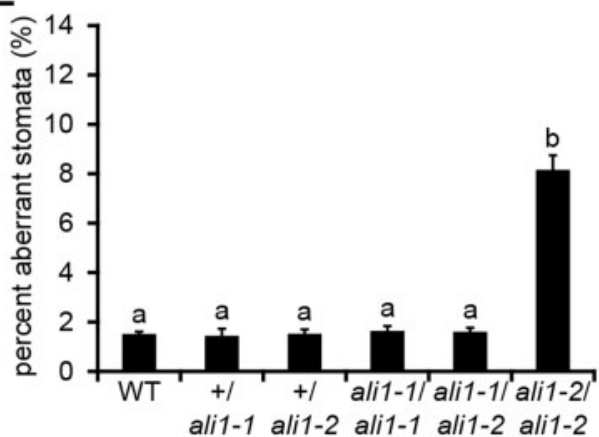

G

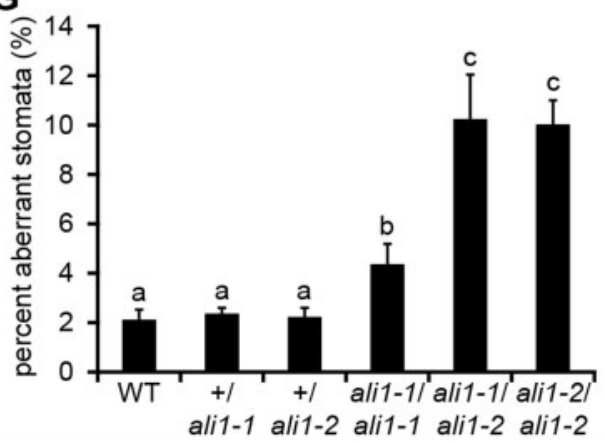

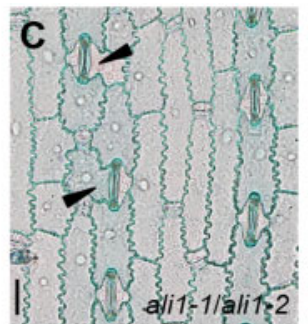

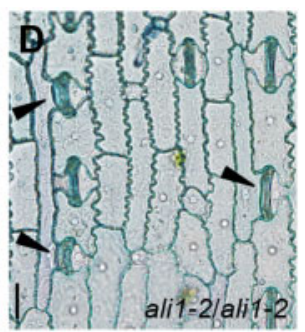

$\mathbf{F}$

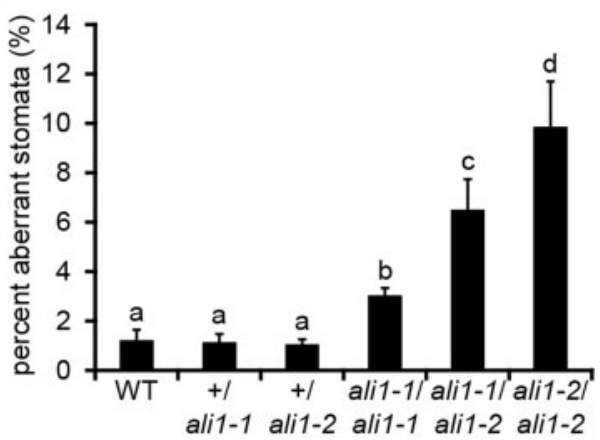

Figure 2 Aberrant stomatal complexes in ali1 mutants. (A-D) Leaf epidermal peels stained with TBO of the $10^{\text {th }}$ leaf of (A) wild-type, (B) ali1-1/ali1-1, (C) ali1-1/ali1-2, and (D) ali1-2/ali1-2 plants at V11 stage in the greenhouse. Black triangles indicate subsidiary cells of aberrant stomata. Scale bars, $20 \mu \mathrm{m}$. $(E-G)$ The percentage of aberrant stomata in wild type $(n=6),+/$ ali1-1 $(n=6),+$ ali1-2 $(n=6)$, ali1-1/ali1-1 $(n=6)$, ali1-1/ali1-2 $(n=6)$, and ali1-2/ali1-2 $(n=6)$ from the (E) $4^{\text {th }}$ leaf at V5 stage or (F) $10^{\text {th }}$ leaf at V11 stage grown in the greenhouse or (G) from the $8^{\text {th }}$ leaf at V9 stage grown in the field. (E-G) Error bars indicated standard deviation. Connecting letter report indicating significance between genotypes determined by ANOVA and post-hoc analysis using the Holm-Sidak algorithm.

divisions in juvenile leaves indicating that the ali1 gene was necessary for normal juvenile stomatal development.

Incomplete dominance between the mutant alleles was also observed for the SMC division phenotypes. Normal SMC divisions were observed on the fourth leaf of ali1-1/ali1-2 plants grown in the greenhouse indicating that the protein encoded by ali1-1 was sufficient to support normal SMC division in juvenile leaves and ali1-1 was dominant to ali1-2 (Figure 3, A-F). A greater proportion of stomatal complexes were aberrant on the eighth leaf of field grown (Figure 3, G-L) and the tenth leaf of greenhouse grown ali11/ali1-2 plants than in ali1-1 homozygous mutants, indicating that a single dose of ali1-1 was insufficient to support normal development after phase change. The ali1-1/ali1-2 heterozygotes were still less severe than the ali1-2 homozygotes in the greenhouse-grown adult tenth leaves indicating the same dosage dependence as observed for height (Figure 1). Interestingly, the SMC division phenotypes of ali1-1/ali1-2 heterozygotes and ali1-2 homozygotes were not different on the eighth leaf of field-grown plants, indicating that ali1-2 was completely dominant to ali1-1 for SMC division in adult leaves in the field but not greenhouse conditions. The ali1-1 mutant plants are suppressed in the greenhouse and the reduction in aberrant stomata of ali1-1/ali1-2 as compared to ali1-2 when grown in the greenhouse is likely due to differences between the two growing environments (Figures 1 and 2). The aberrant SMC divisions were not accompanied by defects in stomatal initiation as there were no significant differences in stomatal indices or stomatal densities (Supplementary Tables S5 and S6). Therefore, the SMC division defect was solely the result of failed asymmetric division and not due to an increased number of guard mother cells or SMCs.

\section{NPC members show a transcriptional compensation effect in ali1-1 mutants}

To investigate the gene expression consequences of reduced ali1 function we performed RNA-seq experiments comparing ali1-1 and wild-type tissues. Tassels were the most severely affected organ in ali1-1 mutants (Figure 1). RNA was extracted from stem tissue in developing tassels just before tassel emergence from the developing leaf whorl from wild-type and mutant plants and differential gene expression was assessed by RNA-seq. Assessment of differential gene expression identified 962 genes significantly up-regulated and 1719 genes down-regulated in ali1-1 tassels as compared to wild type with FDR less than 0.05 and greater than twofold change (Supplementary File S2). The ALADIN1 transcript 

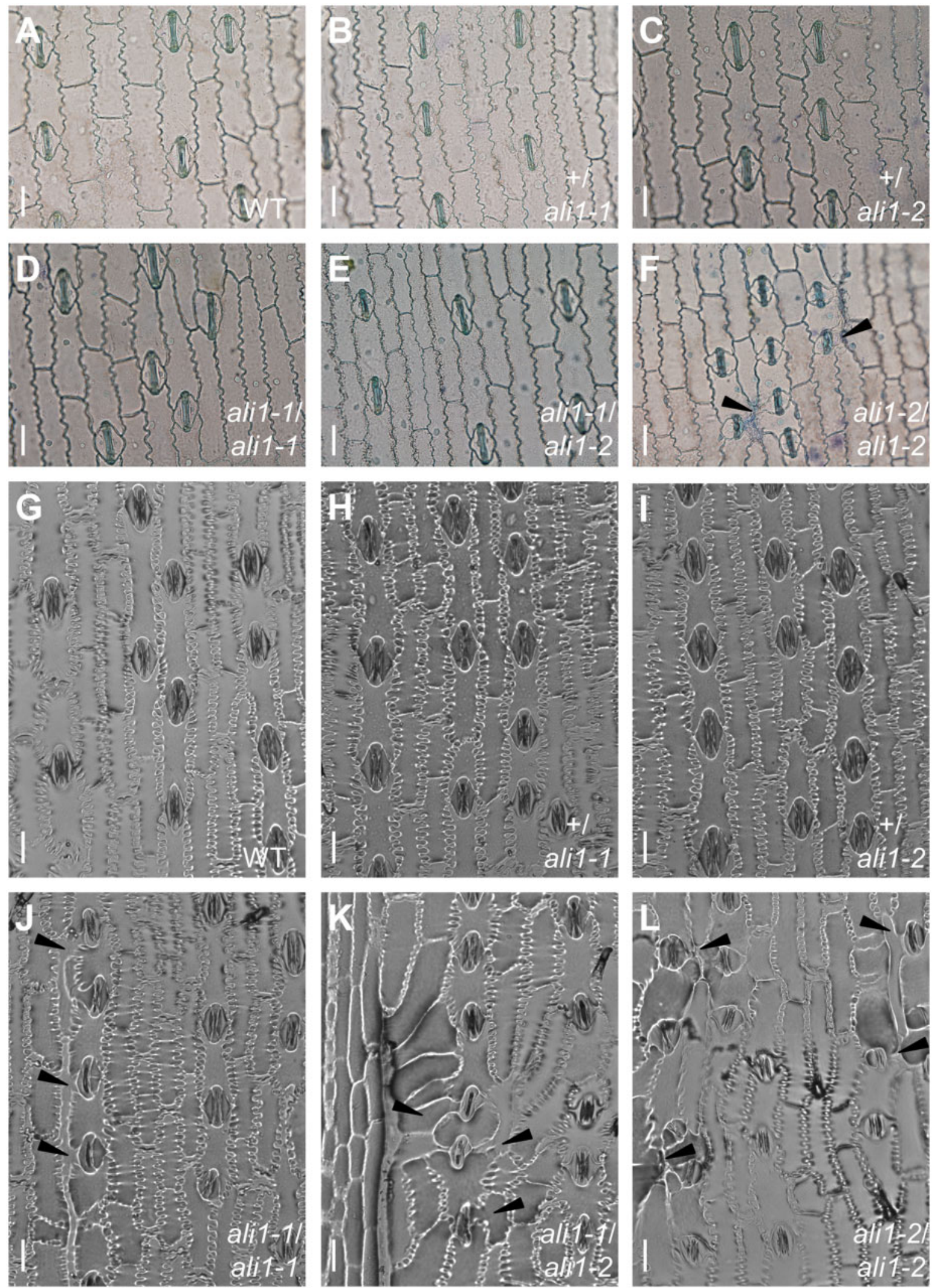

Figure 3 Aberrant stomatal complexes and abnormal cell morphology phenotypes of ali1 mutants. (A-F) Epidermal peels from the 4th leaf abaxial surface at V5 stage of (A) WT, (B) +/ali1-1, (C) +/ali1-2, (D) ali1-1/ali1-1, (E) ali1-1/ali1-2, and (F) ali1-2/ali1-2 plants grown in the greenhouse. (G-L) Epidermal imprints from the 8th leaf abaxial surface at V9 stage of (G) WT, (H) +/ali1-1, (I) +/ali1-2, (J) ali1-1/ali1-1, (K) ali1-1/ali1-2, and (L) ali1-2/ali1-2 plants grown in the field. (A-L) Black triangles indicate subsidiary cells of aberrant stomata. Scale bar, $20 \mu \mathrm{m}$. 
was not differentially expressed in the experiment. The presence of the ALADIN1 transcript in the ali1-1 mutant provides an explanation for apparent partial function of the ali1-1 allele and indicates that the premature stop codon that was still encoded by the last exon did not trigger nonsense-mediated decay of the mRNA. Known determinants of asymmetric division in the SMC, PANGLOSS1 (PAN1) and suppressor of cAMP receptor/WiskottAldrich syndrome protein-family verprolin-homologous protein (SCAR/WAVE) complex member BRICK1 (BRK1) (Gallagher and Smith 2000; Facette et al. 2015), were significantly down-regulated in ali1-1 tassels (Supplementary File S9) suggesting that mis-expression of these genes may contribute to aberrant control of SMC divisions in ali1 mutants. Among the differentially expressed transcripts, only four NPC components, RIBONUCLEIC ACID EXPORT1-LIKE1 (RAE1-L1), CANDIDATE GENE1 (CG1), NUCLEOPORIN205 (NUP205), and CONSTITUTIVE EXPRESSOR OF PR GENES5 (CPR5) were significantly up-regulated in ali1-1 tassels (Figure 4 and Supplementary Table S7). RAE1 and ALADIN from A. thaliana physically interact and CG1 was previously predicted to be in the same subcomplex as ALI1 on the cytoplasmic region of the NPC (Tamura et al. 2010). To assess if other ALADIN-interacting proteins might display compensatory changes in expression in the ali1-1 mutant, genes similar to known ALADIN1interacting proteins from humans were identified. Of the 64 maize sequences similar to the human ALADIN protein interactors, five transcripts were differentially expressed at a false discovery rate (FDR) of $<0.05$ including the C-TERMINAL DOMAIN NUCLEAR ENVELOPE PHOSPHATASE1-L1 (CTDNEP1-L1; up-regulated in ali1-1), RAN BINDING PROTEIN2-L5 (RANBP2-L5; down-regulated in ali1-1), and RANBP2-L7 (down-regulated in ali1-1) genes (Supplementary Table S8). ALADIN has been shown in humans to be anchored to the NPC via NUCLEAR DIVISION CYCLE1 (NDC1) (Kind et al. 2009; Yamazumi et al. 2009; Gu et al. 2016) but a maize homolog of this gene was not differentially expressed in ali1-1 mutants. The NPC has comprised of 30 subunits that have been described in detail in Arabidopsis (Tamura et al. 2010), so we identified the maize genes encoding the homologs of these genes (Figure 4, Supplementary Tables S5 and S6). In developing mutant tassels, the mRNA encoded by NPC component genes were more likely to be accumulated at higher levels (40 of 49 expressed transcripts) in ali1-1 mutant tassel stem tissue than in wild type ( $\chi^{2}$ P-value $=9.49 \mathrm{E}$-06; Figure 4$)$.

Unlike tassels, juvenile leaves were the least phenotypically affected aerial tissues in the ali1 mutants. In an effort to better assess direct effects of ali1-1, we performed RNA-seq using RNA extracted from mutant and wild-type leaves at 15 DAP, before any phenotypes were visible (Figures $2 \mathrm{E}$ and $3 \mathrm{D}$ ). Again, more NPC genes were up-regulated than down-regulated in ali1-1 as compared to wild type (32 of 47 expressed transcripts; $\chi^{2} P$-value = 0.0131; Figure 4, Supplementary Table S9, and Supplementary File S10). However, only 16 genes were significantly up-regulated and 40 genes down-regulated after a transcriptome-wide FDR adjustment and this dropped to 9-up and 14-down when a twofold expression difference threshold was applied (Supplementary File S3). This indicates that the weak ali1-1 allele triggered the accumulation of transcripts encoding NPC components prior to visible effects on plant growth or SMC division defects. Of the maize homologs of ALADIN interactors in human, only RANBP2-L4 was differentially expressed (Supplementary Table S9).

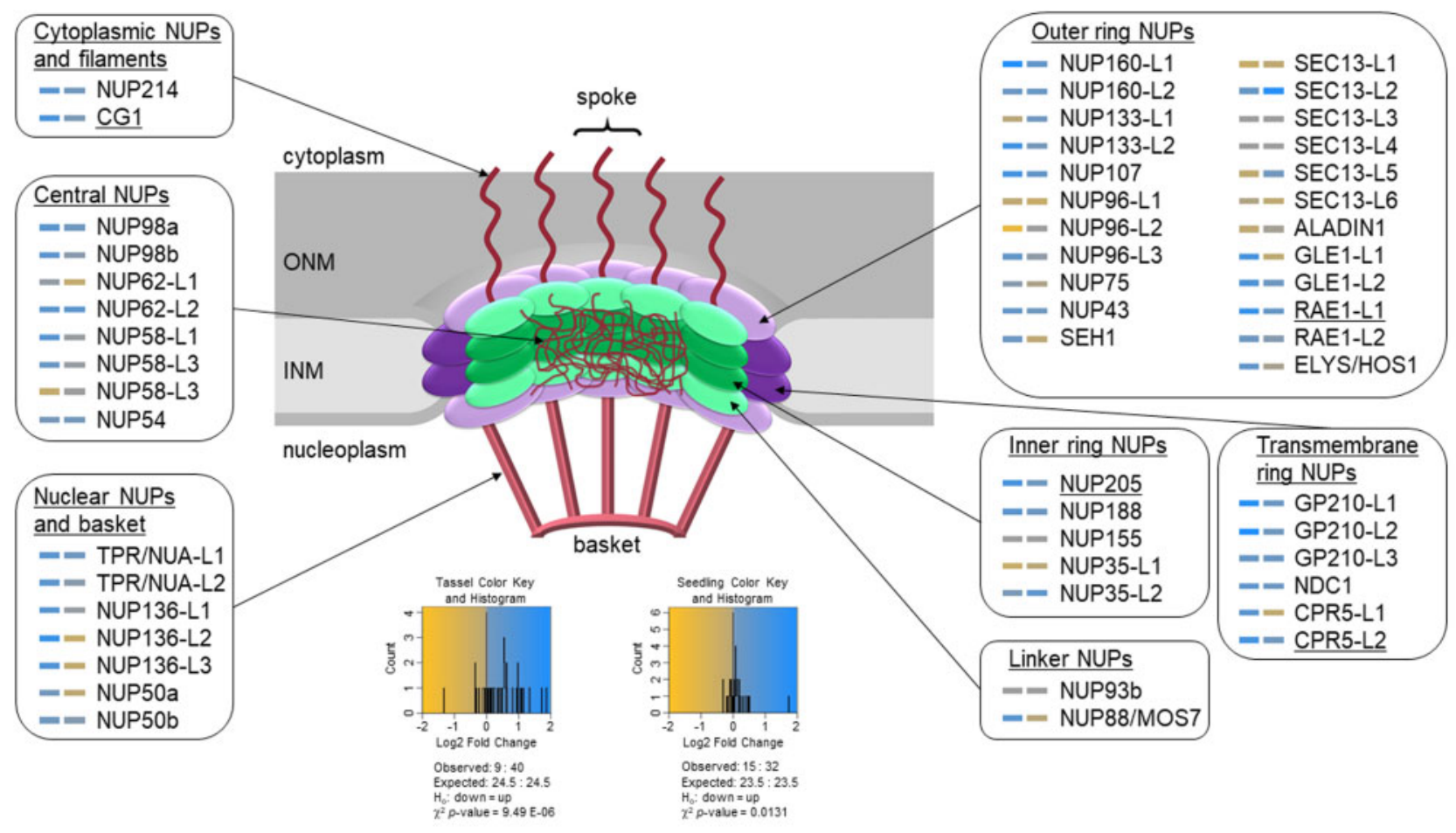

Figure 4 NPC gene expression in ali1-1 mutants. Heatmap of NPC gene expression in ali1-1 tassels (left) and ali1-1 seedlings (right) as compared to respective wild type. Putative NPC genes in maize were identified by BLASTP to NPC members from Arabidopsis (Kind et al. 2009; Tamura et al. 2010; Tamura and Hara-Nishimura 2013; Gu et al. 2016). Outlined gene groups indicate sub-complexes within the NPC as relative location and structure are shown in the middle figure. Gold color indicates down-regulation and blue indicate up-regulation of respective transcript as indicated by the color key. Underlined transcripts (CG1, RAE1-L1, NUP205, and CPR5-L2) were significant at genome-wide Benjamini-Hochberg corrected P <0.05 for ali1-1 tassels (left). The observed and expected down-regulated and up-regulated transcripts in the NPC, hypothesis test, and $\chi^{2}$ P-value are shown below the color key and histogram for both the tassel and seedling data. 


\section{Discussion}

The ali1 mutants, identify a member of the NPC complex as critical for asymmetric division in plants. A similar effect on subsidiary cell asymmetric division was recently observed for the maize linc kash Atsine-like2 (mlks2) mutant in maize; which is a component of the LINKER OF NUCLEOSKELETON AND CYTOSKELETON (LINC) protein complex that spans the nuclear envelope but is not associated with the NPC (Gumber et al. 2019). The MLKS2 transcript was not differentially accumulated in ali1-1 mutants, nor were any other members of the LINC complex, including SUN- and KASH-domain transcripts (Supplementary File S2). The LINC complex also affects nuclear shape in plants (Zhou et al. 2012; Zhou and Meier 2013; Zhou et al. 2015a, b; Meier et al. 2016; Thorpe and Charpentier 2017; Newman-Griffis et al. 2019; Moser et al. 2020) and effects on nuclear shape are observed in the mlks2 mutant (Gumber et al. 2019) but were not observed in ali1-1 mutant leaves. To date, only one mutant in a nuclear pore component, Nup136 in Arabidopsis, controls the shape and size of nuclei (Tamura and Hara-Nishimura 2011). No studies of ALADIN1 homologs in other species identified an effect on nuclear morphology (Huebner et al. 2006, 2004).

The aberrant cell divisions in the ali1 mutants suggest that the NPC can affect nuclear localization or asymmetric phragmoplast assembly at the site of asymmetric divisions. Other mutants of maize, in addition to mlks2, result in aberrant SMC divisions. Among these are mutants in genes encoding components of the actin-like protein-containing SCAR/WAVE complex (Frank et al. 2003; Djakovic et al. 2006; Facette et al. 2015). Interestingly, the SCAR/WAVE component BRK1 mRNA is decreased in ali1 mutants. The brk1 mutant results in a loss of epidermal crenulation, which is more evident in adult leaves, and aberrant SMC divisions. Similarly, PAN1 mRNA is decreased in ali1 (Supplementary Files S2 and S9). The PAN1 protein is localized via the SCAR/WAVE complex and, mutants of pan1 are also affected in asymmetric division and produce aberrant SMC. Given the similar impacts on SMC divisions and the gene expression effects, ali1 may link the SCAR/WAVE complex, required for actin polymerization and cytoskeletal dynamics, to nuclear positioning and asymmetric division control. Exploration of the relationship between ALI1 and cell division dynamics awaits future experimentation in maize as well as in human where these processes may contribute to the etiology of AAAS.

Identification of the weak loss of function ali1-1 allele and the stronger ali1-2 allele allowed us to investigate the dosage-dependent effects on phenotypes. Plant height and SMC division displayed variation in phenotypic expression across an allelic series. The ali1-1 and ali1-2 alleles were completely recessive when in combination with the wild-type allele, indicating that one wild-type copy was sufficient to maintain NPC function. The ali1-1/ali1-2 heterozygote was more severe, for both plant height and SMC asymmetric cell division, than ali1-1/ali1-1 mutants indicating that ali1-1 was haploinsufficient with the ali1-2 allele. These effects were more obvious in adult tissues, adult leaves and stems, and were consistent with an increasing demand for ALI1 after phase change. The observed increase in other phenomena affected by SCAR/WAVE action, such as leaf crenulation, after phase chance may indicate a greater accumulation or role for this protein complex in epidermal development of adult leaves. If this is the case, greater accumulation of ALI1 may be required for stoichiometric protein-protein interactions. This has the capacity to create the threshold effect on SMC divisions at the higher levels of ALI1 activity provided by the ali1-1 mutant homozygotes. Further investigation into these allelic combinations at the protein level and their interactions with determinants of nuclear positioning and the other members of the NPC are needed to test this hypothesis.

Substantial differences were observed for the weak ali1-1 mutant phenotype depending on whether they were grown in the field or the greenhouse. In repeated seasons, and plantings, ali1-1 was substantially suppressed and nearly indistinguishable from wild-type siblings when grown in the greenhouse. Though we do not know the cause of this environmentally contingent phenotype expression in ali1-1 it is reasonable to propose light quality and day length as factors. The dracula2 mutant in Arabidopsis encodes a NUP98 ortholog and is involved in shade-avoidance regulated gene expression (Gallemi et al. 2016). Future investigation into the effects of the shade-avoidance response for ali1 needs to be done.

In this study, we show that the ali1 mutant was encoded by the maize ortholog of the human disease gene AAAS. The ali1-1 allele is encoded by mutation of the codon for the tryptophan at position 430 of the maize protein to a stop. Remarkably, the same position in the human protein (W474) encodes a known disease allele that is also the result of a nonsense mutation (Houlden et al. 2002). The human and mouse genes encode a C-terminal extension (Supplementary Figure S9) but no function of this region has been identified. Deletions of the C-terminal extension after the homologous ali1-1 position that remove all (Q490*) or part (R493*, V497*, or R500*) of the C-terminal extension did not alter protein localization; however, a R478* allele which truncates fewer amino acids than the maize ali1-1 mutation, did result in mis-localized GFP-ALADIN in human cells (Cronshaw and Matunis 2003), and resulted in human disease phenotypes. In humans, this allele has only been clinically observed in heterozygous conditions and results in relatively mild disease in combination with a more severe mutant allele. Given the incomplete dominance observed between ali1-1 and ali1-2 mutants, this suggests that this allele is also haploinsufficient in humans (Houlden et al. 2002). It is unclear if homozygotes at the human W474* allele would exhibit weaker, perhaps even subclinical, phenotypes consistent with AAAS. The dramatic phenotypic impacts, and allelic series, available in maize mirror the situation in humans. Further investigation into the transcriptional and cell biological consequences of ali1-1 and ali1-2 may help identification of the cellular mechanisms responsible for the progressive deleterious effects of mutating ali1 and provide a model system for understanding AAAS in human.

Previously identified Arabidopsis mutants defective in NPC subunits alter nuclear transport (Parry 2014). NPC members also influence gene expression via binding chromatin, changing the chromatin localization in the nucleoplasm, affecting nuclear export of mRNA, and shuttling of proteins (Parry 2015). The increased accumulation of mRNA encoding NPC subunits in ali1-1 mutants suggests the existence of an NPC surveillance mechanism that can increase the abundance of multiple transcripts encoding NPC subunits when the complex was compromised. This effect was visible in ali1-1 mutants at the juvenile stage prior to any visible phenotypic effects suggesting that it was not a secondary response to altered cellular morphology in the mutants. In addition, the lack of a phenotype in juvenile plants may be the result of this compensatory change in NPC subunit expression. If so, a mutagenesis experiment should recover enhancers of the ali1-1 phenotype when this process is disrupted. It remains to be tested if the observed compensation in NPC subunit transcripts in ali1-1 are the result of monitoring for aberrant transport processes. 


\section{Acknowledgments}

The authors would like to thank Jim Beaty and the crew at the Purdue University ACRE for help with field-grown maize used in these studies. This study is presented as a gift on the occasion of Jim Beaty's retirement from Purdue in thanks for his support of graduate student research. They would also like to thank Rob Eddy and the crew at the Purdue University Horticulture Growth Facilities with assistance for greenhouse grown maize used in these studies. Mention of trade names or commercial products in this publication is solely for the purpose of providing specific information and does not imply recommendation or endorsement by the U.S. Department of Agriculture. The U.S. Department of Agriculture is an equal opportunity provider and employer.

\section{Funding}

This work was supported by funds from the National Science Foundation (NSF) to B.P.D. and G.J. (PGRP \# 1444503), B.S. (CAREER \#1054918), and C.W. (PGRP \#1025976). Work by N.B.B. supported by U.S. Department of Agriculture, National Institute of Food and Agriculture (USDA-NIFA) fellowships to N.B.B (NIFA \#2017-67011-26077; \#2019-67012-29655).

\section{Conflicts of interest}

None declared.

\section{Literature cited}

Anders S, Pyl PT, Huber W. 2015. HTSeq-a Python framework to work with high-throughput sequencing data. Bioinformatics. 31: 166-169.

Best NB, Hartwig T, Budka J, Fujioka S, Johal G, et al. 2016. nana plant2 encodes a maize ortholog of the Arabidopsis brassinosteroid biosynthesis gene DWARF1, identifying developmental interactions between brassinosteroids and gibberellins. Plant Physiol. 171: 2633-2647.

Binder A, Parniske M. 2013. The nuclear pore complex in symbiosis and pathogen defence. Ann Plant Rev. 46:229-254.

Bolger AM, Lohse M, Usadel B. 2014. Trimmomatic: a flexible trimmer for Illumina sequence data. Bioinformatics. 30:2114-2120.

Cheng YT, Germain H, Wiermer M, Bi D, Xu F, et al. 2009. Nuclear pore complex component MOS7/Nup88 is required for innate immunity and nuclear accumulation of defense regulators in Arabidopsis. Plant Cell. 21:2503-2516.

Cingolani P, Platts A, Wang Le L, Coon M, Nguyen T, et al. 2012. A program for annotating and predicting the effects of single nucleotide polymorphisms, SnpEff: SNPs in the genome of Drosophila melanogaster strain w1118; iso-2; iso-3. Fly (Austin). 6:80-92.

Cronshaw JM, Krutchinsky AN, Zhang W, Chait BY, Matunis MJ. 2002. Proteomic analysis of the mammalian nuclear pore complex. J Cell Biol. 158:915-927.

Cronshaw JM, Matunis MJ. 2003. The nuclear pore complex protein ALADIN is mislocalized in triple A syndrome. Proc Natl Acad Sci USA. 100:5823-5827.

Djakovic S, Dyachok J, Burke M, Frank MJ, Smith LG. 2006. BRICK1/HSPC300 functions with SCAR and the ARP2/3 complex to regulate epidermal cell shape in Arabidopsis. Development. 133:1091-1100.

Dong CH, Hu X, Tang W, Zheng X, Kim YS, et al. 2006. A putative Arabidopsis nucleoporin, AtNUP160, is critical for RNA export and required for plant tolerance to cold stress. Mol Cell Biol. 26: 9533-9543.,

Eggermont K, Goderis IJ, Broekaert WF. 1996. High-throughput RNA extraction from plant samples based on homogenisation by reciprocal shaking in the presence of a mixture of sand and glass beads. Plant Mol Biol Rep. 14:273-279.

Facette MR, Park Y, Sutimantanapi D, Luo A, Cartwright HN, et al. 2015. The SCAR/WAVE complex polarizes PAN receptors and promotes division asymmetry in maize. Nat Plants. 1:14024.

Fiserova J, Kiseleva E, Goldberg MW. 2009. Nuclear envelope and nuclear pore complex structure and organization in tobacco BY-2 cells. Plant J. 59:243-255.

Fournier C, Andrieu B. 2000. Dynamics of the elongation of internodes in maize (Zea mays L.): Analysis of phases of elongation and their relationships to phytomer development. Ann Bot. 86: 551-563.

Frank MJ, Cartwright HN, Smith LG. 2003. Three Brick genes have distinct functions in a common pathway promoting polarized cell division and cell morphogenesis in the maize leaf epidermis. Development. 130:753-762.

Gallagher K, Smith LG. 2000. Roles for polarity and nuclear determinants in specifying daughter cell fates after an asymmetric cell division in the maize leaf. Curr Biol. 10:1229-1232.

Gallemi M, Galstyan A, Paulisic S, Then C, Ferrandez-Ayela A, et al. 2016. DRACULA2 is a dynamic nucleoporin with a role in regulating the shade avoidance syndrome in Arabidopsis. Development. 143:1623-1631.

Goodstein DM, Shu S, Howson R, Neupane R, Hayes RD, et al. 2012. Phytozome: a comparative platform for green plant genomics. Nucleic Acids Res. 40:D1178-D1186

Gu Y, Zebell SG, Liang Z, Wang S, Kang BH, et al. 2016. Nuclear pore permeabilization is a convergent signaling event in effector-triggered immunity. Cell. 166:1526-1538.

Gumber HK, McKenna JF, Tolmie AF, Jalovec AM, Kartick AC, et al. 2019. MLKS2 is an ARM domain and F-actin-associated KASH protein that functions in stomatal complex development and meiotic chromosome segregation. Nucleus. 10:144-166.

Houlden H, Smith S, Carvalho MD, Blake J, Mathias C, et al. 2002. Clinical and genetic characterization of families with triple A (Allgrove) syndrome. Brain. 125:2681-2690.

Huebner A, Kaindl AM, Knobeloch KP, Petzold H, Mann P, et al. 2004. The Triple A syndrome is due to mutations in ALADIN, a novel member of the nuclear pore complex. Endocr Res. 30: 891-8999.

Huebner A, Mann P, Rohde E, Kaindl AM, Witt M, et al. 2006. Mice lacking the nuclear pore complex protein ALADIN show female infertility but fail to develop a phenotype resembling human triple A syndrome. Mol Cell Biol. 26:1879-1887.

Kind B, Koehler K, Lorenz M, Huebner A. 2009. The nuclear pore complex protein ALADIN is anchored via NDC1 but not via POM121 and GP210 in the nuclear envelope. Biochem Biophys Res Commun. 390:205-210.

Lamesch P, Berardini TZ, Li D, Swarbreck D, Wilks C, et al. 2012. The Arabidopsis Information Resource (TAIR): improved gene annotation and new tools. Nucleic Acids Res. 40:D1202-D1210.

Langmead B, Salzberg SL. 2012. Fast gapped-read alignment with Bowtie 2. Nat Methods. 9:357-359.

Li H, Durbin R. 2009. Fast and accurate short read alignment with Burrows-Wheeler transform. Bioinformatics. 25:1754-1760.

Li H, Handsaker B, Wysoker A, Fennell T, Ruan J, et al. 2009. The Sequence Alignment/Map format and SAMtools. Bioinformatics. 25:2078-2079. 
Lin DH, Stuwe T, Schilbach S, Rundlet EJ, Perriches T, et al. 2016. Architecture of the symmetric core of the nuclear pore. Science. 352:aaf1015.

Liu HL, De Souza CP, Osmani AH, Osmani SA. 2009. The three fungal transmembrane nuclear pore complex proteins of Aspergillus nidulans are dispensable in the presence of an intact An-Nup84-120 complex. Mol Biol Cell. 20:616-630.

Love MI, Huber W, Anders S. 2014. Moderated estimation of fold change and dispersion for RNA-seq data with DESeq2. Genome Biol. 15:550.

Meier I, Griffis AH, Groves NR, Wagner A. 2016. Regulation of nuclear shape and size in plants. Curr Opin Cell Biol. 40:114-123.

Michelmore RW, Paran I, Kesseli RV. 1991. Identification of markers Linked to disease-resistance genes by bulked segregant analysis-a rapid method to detect markers in specific genomic regions by using segregating populations. Proc Natl Acad Sci USA. 88: 9828-9832.

Moser M, Kirkpatrick A, Groves NR, Meier I. 2020. LINC-complex mediated positioning of the vegetative nucleus is involved in calcium and ROS signaling in Arabidopsis pollen tubes. Nucleus. 11: 149-163.

Neff MM, Neff JD, Chory J, Pepper AE. 1998. dCAPS, a simple technique for the genetic analysis of single nucleotide polymorphisms: experimental applications in Arabidopsis thaliana genetics. Plant J. 14:387-392.

Neumann N, Jeffares DC, Poole AM. 2006. Outsourcing the nucleus: nuclear pore complex genes are no longer encoded in nucleomorph genomes. Evol Bioinformatics. 2:23-34.

Newman-Griffis AH, Del Cerro P, Charpentier M, Meier I. 2019. Medicago LINC complexes function in nuclear morphology, nuclear movement, and root nodule symbiosis. Plant Physiol. 179: 491-506.

Nofrini V, Giacomo DD, Mecucci C. 2016. Nucleoporin genes in human diseases. Eur J Hum Genet. 24:1388-1395.

O'Brien KP, Remm M, Sonnhammer EL. 2005. Inparanoid: a comprehensive database of eukaryotic orthologs. Nucleic Acids Res. 33: D476-480.

Parry G. 2014. Components of the Arabidopsis nuclear pore complex play multiple diverse roles in control of plant growth. J Exp Bot. 65:6057-6067.

Parry G. 2015. The plant nuclear envelope and regulation of gene expression. J Exp Bot. 66:1673-1685.

Pryor A. 1993. Transposon tagging of a rust resistance gene in maize. In: Advances in Molecular Genetics of Plant-Microbe Interaction. Dordrecht, The Netherlands: Kluwer Academic Publisher.

Rasala BA, Orjalo AV, Shen ZX, Briggs S, Forbes DJ. 2006. ELYS is a dual nucleoporin/kinetochore protein required for nuclear pore assembly and proper cell division. Proc Natl Acad Sci USA. 103: 17801-17806.

Reichelt R, Holzenburg A, Buhle EL, Jarnik M, Engel A, et al. 1990. Correlation between structure and mass-distribution of the nuclear-pore complex and of distinct pore complex components. J Cell Biol. 110:883-894.
Roth C, and, Wiermer M. 2012. Nucleoporins Nup160 and Seh1 are required for disease resistance in Arabidopsis. Plant Signal Behav. 7:1212-1214.

Rout MP, Aitchison JD, Suprapto A, Hjertaas K, Zhao Y, et al. 2000. The yeast nuclear pore complex: composition, architecture, and transport mechanism. J Cell Biol. 148:635-651.

Sakuma S, D'Angelo MA. 2017. The roles of the nuclear pore complex in cellular dysfunction, aging and disease. Semin Cell Dev Biol. 68:72-84.

Sanger F, Nicklen S, Coulson AR. 1977. DNA sequencing with chain-terminating inhibitors. Proc Natl Acad Sci USA. 74:5463-5467.

Sarkar KR, Coe EH. 1966. A genetic analysis of the origin of maternal haploids in maize. Genetics. 54:453-464.

Stuwe T, Bley CJ, Thierbach K, Petrovic S, Schilbach S, et al. 2015 Architecture of the fungal nuclear pore inner ring complex. Science. 350:56-64.

Tamura K, Fukao Y, Iwamoto M, Haraguchi T, Hara-Nishimura I. 2010. Identification and characterization of nuclear pore complex components in Arabidopsis thaliana. Plant Cell. 22:4084-4097.

Tamura K, Hara-Nishimura I. 2011. Involvement of the nuclear pore complex in morphology of the plant nucleus. Nucleus. 2:168-172.

Tamura K, Hara-Nishimura I. 2013. The molecular architecture of the plant nuclear pore complex. J Exp Bot. 64:823-832.

Tatusova TA, Madden TL. 1999. BLAST 2 Sequences, a new tool for comparing protein and nucleotide sequences. FEMS Microbiol Lett. 174:247-250.

Thorpe SD, Charpentier M. 2017. Highlight on the dynamic organization of the nucleus. Nucleus. 8:2-10.

Weil CF. 2009. TILLING in grass species. Plant Physiol. 149:158-164.

Wiermer M, Cheng YT, Imkampe J, Li M, Wang D, et al. 2012. Putative members of the Arabidopsis Nup107-160 nuclear pore sub-complex contribute to pathogen defense. Plant J. 70:796-808.

Yamazumi Y, Kamiya A, Nishida A, Nishihara A, Iemura S, et al. 2009. The transmembrane nucleoporin NDC1 is required for targeting of ALADIN to nuclear pore complexes. Biochem Biophys Res Commun. 389:100-104.

Zhang Y, Li X. 2005. A putative nucleoporin 96 Is required for both basal defense and constitutive resistance responses mediated by suppressor of npr1-1,constitutive 1. Plant Cell. 17:1306-1316.

Zhou X, Graumann K, Evans DE, Meier I. 2012. Novel plant SUN-KASH bridges are involved in RanGAP anchoring and nuclear shape determination. J Cell Biol. 196:203-211.

Zhou X, Meier I. 2013. How plants LINC the SUN to KASH. Nucleus. 4 206-215.

Zhou X, Graumann K, Meier I. 2015a. The plant nuclear envelope as a multifunctional platform LINCed by SUN and KASH. J Exp Bot. 66:1649-1659.

Zhou X, Groves NR, Meier I. 2015. Plant nuclear shape is independently determined by the SUN-WIP-WIT2-myosin XI-i complex and CRWN1. Nucleus. 6:144-153.

Communicating editor: R. Rellan-Alvarez 